\title{
Performance Characteristics of Asphalt Binders containing Sodium-Alginate Hollow Fibers and Recycled Materials
}

\author{
Max A. Aguirre ${ }^{1}$, Marwa M. Hassan ${ }^{1, *}$, Sharareh Shirzad ${ }^{1}$, Louay N. Mohammad ${ }^{2}$, Samuel B. Cooper, Jr. ${ }^{3}$, and Ioan I. \\ Negulescu ${ }^{4}$ \\ ${ }^{1}$ Department of Construction Management, Louisiana State University \\ ${ }^{2}$ Department of Civil and Environmental Engineering, Louisiana State University \\ ${ }^{3}$ Department Louisiana Transportation Research Center \\ ${ }^{4}$ Department of Chemistry, Louisiana State University
}

\begin{abstract}
Self-healing products such as hollow-fibers filled with an asphalt rejuvenator present an emerging technology that would enhance an asphalt mixture's resistance to cracking damage. The objective of this study was to evaluate the rejuvenating efficiency of sodium-alginate fibers containing a rejuvenator product using asphalt binder blends containing extracted binder from recycled materials. The effects of adding extracted binder from recycled materials and sodium-alginate fibers on asphalt binder blends were evaluated by conducting a series of chemical and binder tests. HP-GPC and FTIR test results showed that the addition of fibers in blends containing recycled materials resulted in an increase in the HMW/LMW ratios. Some of the added polymeric fibers are thought to have increased the HMW fraction, thus leading to increase in the HMW/LMW ratio. The increase of the HMW fraction suggests that some of the fibers, which are polymers, caused the increase in the HMW/LMW ratios. MSCR test results showed that a binder blend with extracted binder from recycled materials and sodium-alginate fibers would have less rutting susceptibility than a conventional virgin binder would.
\end{abstract}

\section{Introduction}

Asphalt rejuvenating agents have emerged as a solution to improve the life expectancy of asphalt mixtures containing recycled materials by increasing the blending between aged and virgin asphalt binders. Asphalt rejuvenators include cationic emulsions containing maltenes, which are used to deposit the blend of maltene fractions on the films of aged binder [1]. Thus, the addition of maltene fractions might reverse the aging process of asphalt binder by restoring the maltene-toasphaltene ratio [2]. However, spreading rejuvenator on the pavement surface has negative effects such as loss of the skid resistance and poor penetration into the pavement structure [3].

The encapsulation of a rejuvenator product has emerged as an innovative approach to disperse the rejuvenator product into the asphalt mix. An encapsulation mechanism is used in sodium-alginate hollow fibers filled with a rejuvenator, which consists of a polymer shell and a rejuvenating product as the core material. It is a promising approach as it would enhance the distribution of the rejuvenating product, and thus restoring the original properties of the aged binder in the recycled materials. Furthermore, the elastic property of polymer shell could potentially improve the performance of asphalt binder and asphalt mixtures against common distresses such as permanent deformation and cracking at low and intermediate temperature.

\section{Objectives}

The objectives of this study were the following: (1) Evaluate the rheological properties of asphalt binder blends with sodium-alginate hollow fibers filled with a rejuvenator through laboratory tests; and (2) Assess the molecular/fractional compositions of binder blends with hollow fibers through a series of chemical analyses.

\section{Background}

\subsection{Asphalt rejuvenation}

Asphalt rejuvenator products include low viscosity oil with high maltenes content, which has been shown to be the most effective treatment to partially restore asphalt properties [4]. A study evaluated the effect of asphalt rejuvenators on a binder classified as PG $58-22$ by conducting a series of laboratory tests [5]. The study observed a softening effect with the addition of the evaluated rejuvenators, which resulted in a decrease in viscosity, increase in rutting susceptibility and enhancement against cracking. Similarly, another study concluded that the addition of rejuvenator products in

\footnotetext{
* Corresponding author: marwa@1su.edu
} 
asphalt mixtures containing recycled materials resulted in an improvement in the fatigue cracking resistance compared to conventional mixtures [6]. The softening effect of asphalt rejuvenators was also reported in a study conducted by Aguirre et al. where the addition of asphalt rejuvenators resulted in an increase in the rut depth of asphalt mixtures at high-temperature [7].

\subsection{Encapsulation of asphalt rejuvenator products}

The idea of encapsulating a rejuvenator product is to control the release of the core material by controlling the permeability of the shell material. A technique to encapsulate an asphalt rejuvenator is the synthesis of hollow-fibers containing a rejuvenator product as core material. Tabakovic et al. [8] developed compartment alginate fibers containing a rejuvenator as a healing agent delivery, as well as a healing triggering mechanism for asphalt pavements [8]. Furthermore, Aguirre et al. conducted an optimization process on the production parameters of sodium-alginate fibers containing a bio-oil product as core material as selfhealing and rejuvenating agents for aged asphalt binder [9]. In addition, the study found that $5 \%$ fiber content by weight of virgin binder was the optimum fiber content to enhance rheological properties of binder blends containing extracted binder from recycled materials.

\section{Experimental program}

\subsection{Evaluation of the rheological properties of asphalt binder blends with fibers}

The objective of the binder experiment was to evaluate the effects of adding sodium-alginate fibers on asphalt binder blends containing recycled materials. The experimental test matrix is shown in Table 1. An unmodified binder, i.e. PG 64-22, was selected to evaluate the effect of adding the synthesized fibers. In addition, extracted binder from recycled materials (RAS and RAP) was incorporated in the selected binder blends at 5 and $20 \%$ by weight of the virgin binder, respectively. The asphalt binder blends shown in Table 1 were prepared by mixing virgin binder with the prepared fibers and extracted binder at a temperature of $163^{\circ} \mathrm{C}$. The different asphalt blends were prepared by using a high-shear mixer rotating at a speed of 3,600 rpm for 30 minutes to achieve good mixing and dispersion of the fibers and extracted binder in the different asphalt binder blends.

\subsubsection{Performance grading (PG grading)}

Rheological tests such as Dynamic Shear Rheometer (DSR) and Bending Beam Rheometer (BBR) were used to assess the rheological properties of the asphalt binder blends shown in Table 1. PG grading was performed in accordance with AASHTO M 320 [10].

\subsubsection{Multiple stress creep recovery (MSCR)}

The MSCR test was conducted to characterize the rutting susceptibility of the different asphalt binder blends. MSCR test consists of applying creep and recovery periods and to measure the percentage of recovery and non-recoverable creep compliance $\left(\mathrm{J}_{\mathrm{nr}}\right)$. The MSCR was performed in accordance to AASHTO TP 70 at a testing temperature of $67^{\circ} \mathrm{C}[11]$.

\subsubsection{Linear amplitude sweep (LAS)}

The fatigue resistance of the asphalt blends was characterized by conducting the LAS test. The LAS tests consisted of applying cyclic loading employing systematic, linearly increasing load amplitudes. The LAS was performed in accordance with AASHTO TP 101 [12] in samples aged using RTFO and PAV to simulate the aging of in-service asphalt pavements. LAS test was conducted to determine two fatigue parameters (" $A$ " and "B") based on the asphalt binder fatigue law $\left(N_{f}=\right.$ $\left.A x_{\text {Ymax }}{ }^{B}\right)$. The LAS test consisted of two steps: (1) a frequency sweep test at a low strain amplitude of $0.1 \%$ is used to obtain undamaged material properties (parameter "B" of fatigue law); and (2) an amplitude sweep test with a series of cyclic loading at linearly increasing strain amplitudes at a constant frequency of $10 \mathrm{~Hz}$ is used to determine the parameter " $A$ " of the fatigue law through viscoelastic continuum damage (VECD) mechanics analysis.

Table 1. Test matrix for evaluation of the effects of adding sodium-alginate fibers in asphalt binder blends.

\begin{tabular}{|c|c|c|c|c|}
\hline Blend ID & $\begin{array}{c}\text { Asphalt } \\
\text { Binder }\end{array}$ & $\begin{array}{c}\text { RAS } \\
\text { Content }\end{array}$ & $\begin{array}{c}\text { RAP } \\
\text { Content }\end{array}$ & $\begin{array}{c}\text { Fiber } \\
\text { Content } \\
\text { (\%) }\end{array}$ \\
\hline 64CO & PG 64-22 & - & - & - \\
\hline 64PG3F & PG 64-22 & - & - & $3 \%$ \\
\hline 64PG5F & PG 64-22 & - & - & $5 \%$ \\
\hline 64PG10F & PG 64-22 & - & - & $10 \%$ \\
\hline 64PG5P & PG 64-22 & $\begin{array}{c}5 \% \\
\text { PCWS }\end{array}$ & - & - \\
\hline 64PG5P5F & PG 64-22 & $\begin{array}{c}5 \% \\
\text { PCWS }\end{array}$ & - & $5 \%$ \\
\hline 64PG20RAP & PG 64-22 & - & $20 \%$ & - \\
\hline 64PG20RAP5F & PG 64-22 & - & $20 \%$ & $5 \%$ \\
\hline 64PG5P20RAP & PG 64-22 & $\begin{array}{c}5 \% \\
\text { PCWS }\end{array}$ & $20 \%$ & - \\
\hline 64PG5P20RAP5F & PG 64-22 & $\begin{array}{c}5 \% \\
\text { PCWS }\end{array}$ & $20 \%$ & $5 \%$ \\
\hline
\end{tabular}

\subsection{Chemical Analysis of Asphalt Binder Blends with Fibers}

\subsubsection{High-pressure gel permeation chromatography}

High Pressure Gel Permeation Chromatography (HPGPC) was performed using an EcoSEC system (HLC8320GPC) of Tosoh Corporation, equipped with a differential refractive index detector (RI) and UV detector. A set of four micro-styragel columns of pore 
sizes $200 \AA, 75 \AA$ ( 2 columns) and $30 \AA$ from Tosoh Bioscience was used in the analysis. Tetrahydrofuran (THF) at a flow rate of $0.35 \mathrm{ml} / \mathrm{min}$. was used as the solvent. Columns were calibrated using polystyrene standard mixtures PStQuick B (MW= 5480000, 706000, 96400, 10200, and 1000 daltons), PStQuick E (MW= 355000, 37900, 5970, and 1000 daltons), and PStQuick $\mathrm{F}(\mathrm{MW}=190000,18100,2500$, and 500 daltons) from Tosoh Bioscience. Filtered solutions prepared with the binder blends and THF solvent were processed through a 0.45-micron Teflon filters prior to running the HP-GPC test analysis. The concentration of asphalt solution was $0.5 \%$.

\subsubsection{Fourier transform infrared spectroscopy (FTIR)}

FTIR spectra were obtained using a diamond single reflection attenuated total reflectance (ATR) instrument (Bruker Optics alpha) with the following settings for data collection: 32 scans/sample, spectral resolution 4 $\mathrm{cm}^{-1}$, wave number range $4000-500 \mathrm{~cm}^{-1}$. A few drops of the GPC asphalt solution ( $0.5 \%$ in THF) was placed on the ATR crystal plate and the solvent allowed evaporating. The spectrum was collected after the complete evaporation of the solvent. FTIR spectra of the aged samples show a peak around $1700 \mathrm{~cm}^{-1}$, which is the characteristic of $\mathrm{C}=\mathrm{O}$ species. The carbonyl index was calculated from the band areas measured from valley to valley [13] according to Equation (1). This was accomplished using the OPUS spectroscopy software provided with the Bruker FTIR instrument.

Carbonyl
Index
$($ ICO) $\quad \frac{\text { Area around } 1700 \mathrm{~cm}^{-1}}{\text { Area around } 1460 \mathrm{~cm}^{-1} \& \text { Area }}$

\section{Results and analysis}

\subsection{Chemical Composition of Binder Blends with Fibers}

\subsubsection{Molecular weight distribution}

HP-GPC was used to measure asphalt blends molecular weight distributions, i.e. the percentage of asphaltenes to maltenes that are present in the binder blends. Determining the percentage of asphaltenes and maltenes before and after blending the virgin binders with recycled materials and fibers provided information about the efficiency of the fibers to act as a rejuvenator. The HP-GPC results are shown in Table 2. Table 2 shows that the extracted binders from RAS and RAP had the highest high-molecular weight/ low-molecular weight ratio (HMW/LMW) among the evaluated binder blends. Furthermore, it is shown in Table 2 that the addition of sodium-alginate fibers resulted in an increase in the HMW/LMW ratio. The increase of the HMW fraction suggests that some of the fibers, which are polymers, caused the increase in the HMW/LMW ratios.
Table 2. Chemical composition of evaluated binder blends.

\begin{tabular}{|c|c|c|c|c|}
\hline Sample & $\begin{array}{c}\text { Others } \\
(\mathbf{\%})\end{array}$ & $\begin{array}{c}\text { HMW } \\
\mathbf{( \% )}\end{array}$ & $\begin{array}{c}\text { LMW } \\
(\mathbf{\%})\end{array}$ & $\begin{array}{c}\text { HMW/LMW } \\
\text { Ratio }\end{array}$ \\
\hline 64CO & 0.52 & 17.35 & 82.13 & 0.21 \\
\hline 64PG5P & 1.82 & 23.63 & 74.55 & 0.32 \\
\hline 64PG6P5F & 1.9 & 24.8 & 73.3 & 0.34 \\
\hline 64PG20RAP & 3.44 & 25.35 & 71.21 & 0.36 \\
\hline 64PG20RP5F & 3.33 & 23.46 & 70.89 & 0.33 \\
\hline 64PG5P0RAP & 3.83 & 23.94 & 72.23 & 0.33 \\
\hline 64PG5P20RAP5F & 4.14 & 24.61 & 71.2 & 0.35 \\
\hline RAS & 8.1 & 26.69 & 65.21 & 0.41 \\
\hline RAP & 13.31 & 30.31 & 56.38 & 0.54 \\
\hline \multicolumn{5}{|r}{}
\end{tabular}

\subsubsection{Characterization of oxidative asphalt aging}

The carbonyl Index (ICO) was calculated to evaluate the formation of carbonyl molecules in the binder blends, which is related to the oxidation process in the aging process of asphalt binders. Figure 1 shows that the addition of extracted binder from either RAS and/or RAP resulted in an increase in the ICO index. Also, it is shown that the addition of fibers in blends containing recycled material resulted in an increase in the ICO, which suggests that the rejuvenating product facilitates the extraction of aged binder from the recycled materials and, thus, increased the asphaltene content resulting in stiffer blends.

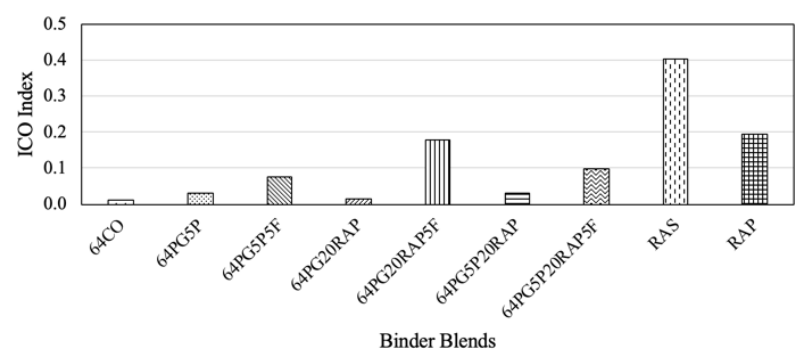

Fig. 1. ICO aging index for binder PG 64-22.

\subsection{Rejuvenation of Asphalt Binders with Sodium-Alginate Fibers}

\subsubsection{PG grading of binder blends}

Table 3 shows that the addition of the hollow fibers did not change the PG grade of the binder blends prepared with PG 64-22 except for 64PG5P20RAP5F, which was the stiffest binder blend. The PG grading of blend 64PG5P20RAP5F could be correlated with the HP-GPC test results as this blend had the second highest HMW/LMW ratio for blends prepared with PG 64-22 shown on Table 2. However, the continuous PG showed a reduction in the HTPG for the binder blends containing the hollow fibers, which may indicate softening of the aged binders. Also, it was noted in the continuous PG grading that the LTPG improved in the binder blends with sodium-alginate fibers, which may indicate an improvement in the low-temperature performance of the binder blends containing only extracted binder from recycled materials. 
Table 3. Summary of PG grading results for binder PG 64-22.

\begin{tabular}{|c|c|c|}
\hline Binder Blend & PG-Grading & $\begin{array}{c}\text { Continuous } \\
\text { PG-Grading }\end{array}$ \\
\hline 64CO & $64-22$ & $68-23.6$ \\
\hline 64PG3F & $64-22$ & $68-24.4$ \\
\hline 64PG5F & $64-22$ & $69.5-23.8$ \\
\hline 64PG10F & $64-22$ & $67-23.7$ \\
\hline 64PG5P & $70-22$ & $71.8-22.3$ \\
\hline 64PG5P5F & $70-22$ & $70.3-22.6$ \\
\hline 64PG20RAP & $76-16$ & $81.8-16.8$ \\
\hline 64PG20RAP5F & $76-16$ & $80.8-19.4$ \\
\hline 64PG5P20RAP & $76-16$ & $81.8-18.0$ \\
\hline 64PG5P20RAP5F & $82-16$ & $82.4-18.0$ \\
\hline
\end{tabular}

\subsubsection{Multiple stress creep recovery}

The MSCR test results for binder PG 64-22 are shown in Table. The binder blends containing extracted binder from recycled materials showed a decrease in the nonrecoverable creep compliance and an increase in the percentage recovery, which indicate an improved rutting resistance compared to the virgin binder PG 64-22. Also, a softening effect from the release of the rejuvenating product from the hollow fibers was observed in Table 4 as noted from the increase in the non-recoverable creep compliance. The reduction in the percentage recovery of the blends containing sodium-alginate fibers could be related to the lack of time to fully recover due to the time-dependent behavior of asphalt binders. Overall, MSCR test results suggest that the addition of sodiumalginate fibers and extracted binder from recycled materials resulted in an improved performance against permanent deformation compared to the conventional virgin binder.

Table 4. MSCR test results for binder PG 64-22.

\begin{tabular}{|c|c|c|c|c|c|}
\hline \multirow{3}{*}{$\begin{array}{l}\text { Asphalt Binder } \\
\text { Blends }\end{array}$} & \multicolumn{5}{|c|}{ MSCR } \\
\hline & \multirow{2}{*}{$\begin{array}{c}\mathbf{J}_{\mathrm{nr} 0.1} @ \\
\mathbf{6 7}{ }^{\circ} \mathbf{C}, \\
\mathrm{kPa}^{-1}\end{array}$} & \multirow{2}{*}{$\begin{array}{c}\mathbf{J}_{\text {nr3.2 }} @ \\
67^{\circ} \mathbf{C}, \\
\mathbf{k P a}^{-1}\end{array}$} & \multirow[b]{2}{*}{$\begin{array}{c}\% J_{n r} \\
\text { diff }\end{array}$} & \multicolumn{2}{|c|}{ \% Recovery } \\
\hline & & & & \begin{tabular}{|l|} 
Stress, \\
$0.1 \mathrm{kPa}$
\end{tabular} & \begin{tabular}{|c|} 
Stress, \\
$3.2 \mathrm{kPa}$
\end{tabular} \\
\hline $64 \mathrm{CO}$ & 3.47 & 3.77 & 8.65 & 1.62 & -0.50 \\
\hline 64PG3F & 3.76 & 4.11 & 9.15 & 1.26 & -0.72 \\
\hline 64PG5F & 3.63 & 4.00 & 10.49 & 1.85 & -0.65 \\
\hline 64PG10F & 4.36 & 4.85 & 11.25 & 1.44 & -1.04 \\
\hline 64PG5P & 2.32 & 2.60 & 12.31 & 4.86 & 0.58 \\
\hline 64PG5P5F & 2.54 & 2.89 & 13.90 & 4.99 & 0.42 \\
\hline 64PG20RAP & 0.43 & 0.49 & 15.00 & 21.80 & 13.59 \\
\hline 64PG20RAP5F & 0.51 & 0.59 & 43.12 & 20.85 & 11.86 \\
\hline 64PG5P20RAP & 0.45 & 0.51 & 15.93 & 22.36 & 13.17 \\
\hline 64PG5P20RAP5F & 0.38 & 0.45 & 16.18 & 24.41 & 14.82 \\
\hline
\end{tabular}

\subsubsection{Linear amplitude sweep}

Table 5 shows that the addition of 5 and $10 \%$ fiber content in virgin binder enhanced the elastic properties of virgin binder PG 64-22 as the "A" parameter increased. Also, it is shown that the addition of aged binder from either RAS or RAP would result in a binder blend more susceptible to cracking as a decrease in the "A" parameter was observed for blends 64PG5P, 64PG20RAP and 64PG5P20RAP compared to the virgin binder. However, the addition of the hollow fibers partially reversed the negative impact of adding an aged binder and the ability of binder blends 64PG5P5F and 64PG20RAP5F to resist fatigue damage. Yet, Table 5 shows that the addition of fibers did not have a pronounced effect on improving the susceptibility of blends to a change in strain levels as the calculated "B" parameter was equal to or higher than the virgin binder PG 64-22.

Table 5. LAS test results for binder PG 64-22.

\begin{tabular}{|c|c|c|}
\hline Asphalt Binder Blends & A-Parameter & B-Parameter \\
\hline 64CO & 16713.6 & 3.9 \\
\hline 64PG3F & 12186.9 & 3.8 \\
\hline 64PG5F & 19271.3 & 3.8 \\
\hline 64PG10F & 57258.2 & 3.7 \\
\hline 64PG5P & 9329.4 & 4.2 \\
\hline 64PG5P5F & 38529.8 & 4.1 \\
\hline 64PG20RAP & 2441.2 & 5.3 \\
\hline 64PG20RAP5F & 5508.7 & 5.1 \\
\hline 64PG5P20RAP & 9986.2 & 4.9 \\
\hline 64PG5P20RAP5F & 4302.6 & 5.6 \\
\hline
\end{tabular}

\section{Summary and conclusions}

The objectives of this study were to evaluate the rheological properties of asphalt binder blends prepared with hollow fibers through laboratory tests, and to assess the chemical compositions of binder blends with fibers through a series of chemical analysis. The following findings and conclusions are drawn based on the results of the experimental program.

\subsection{With respect to the effects of fibers on the chemical compositions of asphalt binder:}

- HP-GPC test results showed that the addition of fibers in blends containing recycled materials resulted in an increase in the HMW/LMW ratio. The increase of the asphaltene fraction suggests that some fibers were broken during the blending process, which released the core material and facilitated the blending between aged and virgin binder in the blends.

- FTIR results showed that the addition of extracted binder from either RAS and/or RAP resulted in an increase in the ICO index. The addition of fibers in blends containing recycled material also resulted in an increase in the ICO, which suggests that the rejuvenating product facilitates the extraction of aged binder from the recycled materials and thus, increased the asphaltene content resulting in stiffer blends.

\subsection{With respect to the effects of the fibers on the rheological properties of asphalt binder:}

- Rheological properties of the binder blends containing recycled materials and sodium-alginate fibers suggested that the fibers did not have a noticeable effect on the final PG grade. 
- MSCR test results showed that a binder blend with extracted binder from recycled materials and sodium-alginate fibers would have less rutting susceptibility than a conventional virgin binder.

- LAS test results showed that the addition of fibers in virgin binders resulted in a increased in the "A" parameter of the fatigue law except with $10 \%$ fiber content in binder PG 64-22.

- LAS test results showed that the addition of fibers in virgin binders resulted in a increased in the "B" parameter of the fatigue law, which would result in lower fatigue resistance.

The authors would also like to acknowledge the laboratory support from the Louisiana Transportation Research Center (LTRC). This study is part of the project 17BSLU06 funded by the Transportation Consortium of South-Central States (Tran-SET).

\section{References}

1. Brownride, J. The role of an asphalt rejuvenator in pavement preservation: use and need for asphalt rejuvenation. Tricor Refining, LLC.

2. Cooper, S., Mohammad, L.N., and Elseifi, M.A. (2014). Laboratory Performance of Asphalt Mixtures Containing Recycled Asphalt Shingles. Journal of the Transportation Research Board, 2445, 94-102.

3. Chiu, C. and Lee, M. (2006). Effectiveness of seal rejuvenators for bituminous pavement surfaces. J. Test. Eval., 34, 390-394.

4. Karlsson, R., and Isacsson., U. (2006). Materialrelated aspects of asphalt recycling-state of the art. Journal of Materials in Civil Engineer, 18.

5. Booshehrian, A., Mogawer, W.S., and Vahidi, S. (2013). Evaluating the Effect of Rejuvenators on the Degree of Blending and Performance of High RAP, RAS, RAP/RAS Mixtures. Road Materials and Pavement Design.

6. Shen, J., Amirkhanian, S., and Aune Miller, J. (2007). Effects of rejuvenating agents on Superpave mixtures containing reclaimed asphalt pavement. Journal of Materials in Civil Engineering, 19(5), 376-384.

7. Aguirre, M.A., Hassan, M.M., Shirzad, S., Mohammad, L.N., and Cooper, S.B. (2017). Performance of asphalt rejuvenators in hot-mix asphalt containing recycled asphalt shingles. Transportation Research Record, 2633, 108-116.

8. Tabakovic, A., Schuyffel, L., Karac, A., and Schlangen, E. (2017). An evaluation of the efficiency of compartmented alginate fibers encapsulating a rejuvenator as an asphalt pavement healing system. Applied Science, 7(647).

9. Aguirre, M.A., Hassan, M.M., Shirzad, S., Cooper Jr., S., Negulescu, I., and Mohammad, L.N. (2018). Evaluation of Hollow-Fibers Encapsulating a Rejuvenator in Asphalt Binders with Recycled Asphalt Shingles. Under Review.
10. AASHTO (2015). Standard specification for performance-graded for asphalt binder. AASHTO M320, Washington, DC.

11. AASHTO (2013). Standard Method of Test for Multiple Stress Creep Recovery (MSCR) Test of Asphalt Binder Using a Dynamic Shear Rheometer (DSR). AASHTO TP 70, Washington, DC.

12. AASHTO (2016). Standard Method of Test for Estimating Fatigue Resistance of Asphalt Binders Using the Linear Amplitude Sweep. AASHTO.

13. Liu, G., Nielsen, E., Komacka, J., Leegwater, G., and Ven, M. (2015). Influence of the soft bitumen on the chemical and rheological properties of reclaimed polymer-modified binders from the "old" surface-layer asphalt. Construction Building Material, 79, 129-135. 